\title{
Interhemispheric magnetic conjugacy
}

\author{
N. Yu. Ganushkina, ${ }^{1,2}$ M. V. Kubyshkina, ${ }^{3}$ N. Partamies, ${ }^{1}$ and E. Tanskanen ${ }^{1}$ \\ Received 9 October 2012; revised 7 December 2012; accepted 16 January 2013; published 4 March 2013.
}

[1] The concept of the inter-hemispheric magnetic conjugacy was investigated by comparing the conjugate points in the northern and southern hemispheres using the Tsyganenko T02 magnetic field model together with the International Geomagnetic Reference Field model for the internal magnetic field to follow the magnetic field lines. We studied the influence of the dipole tilt angle, solar wind (solar wind dynamic pressure and flow direction), and interplanetary magnetic field (IMF) parameters (IMF $B_{y}$ and $B_{z}$ ) on the latitudinal and longitudinal differences between the foot points of magnetic field lines in both hemispheres. It was found that the dominant difference up to $30^{\circ}$ is longitudinal, while latitudinal differences are about $2^{\circ}$. The largest differences are observed at dawn and dusk magnetic local times (MLTs) for large dipole tilt angles during high solar wind dynamic pressure (16-20 nPa) and large IMF $B_{y}$ values ( -15 and $\left.15 \mathrm{nT}\right)$. The asymmetry of conjugate points is present due to nonzero values of IMF $B_{y}$, but there is no real dependence on the magnitude of IMF $B_{y}$. The influence of IMF $B_{z}$ on the interhemispheric conjugacy depends on the sign of the IMF $B_{z}$ but not much on the magnitude. The rotation of the tail current sheet from the Sun-Earth line by several degrees resulted in latitudinal differences of $1^{\circ}$ and longitudinal differences of $15^{\circ}$ at dawn and dusk MLTs for equinox. Testing the concept of magnetic conjugacy with previously reported auroral event observed at Tjörnes (Iceland) and Syowa (Antarctica) observatories confirmed the importance of taking into account the solar wind flow direction, especially when it deviates from radial by more than $2^{\circ}$

Citation: Ganushkina, N. Yu., M. V. Kubyshkina, N. Partamies, and E. Tanskanen (2013), Interhemispheric magnetic conjugacy, J. Geophys. Res. Space Physics, 118, 1049-1061, doi:10.1002/jgra.50137.

\section{Introduction}

[2] Understanding of the magnetic mapping in different conditions and between different regions of the near-Earth space has become an increasingly interesting topic. This is particularly important now during the era of clustered satellite missions (e.g., Cluster and THEMIS), which aim at studying ionospheric signatures of processes at the distances of $10-20 R_{E}$ down in the magnetotail, or when studying conjugate signatures of the aurora with ground-based instruments on both hemispheres.

[3] The basic temporal variation of geomagnetically conjugate points was already described by Ono [1987], who examined the excursion of the conjugate point of the Antarctic Syowa station over Iceland in the northern hemisphere during 30 years. The study was based on tracing

\footnotetext{
${ }^{1}$ Finnish Meteorological Institute, Helsinki, Finland

${ }^{2}$ Department of Atmospheric, Oceanic and Space Sciences, University of Michigan, Ann Arbor, Michigan, USA.

${ }^{3}$ Institute of Physics, University of St. Petersburg, St. Petersburg, Russia.

Corresponding author: N. Yu. Ganushkina, Department of Atmospheric, Oceanic and Space Sciences, University of Michigan, 2455 Hayward St. Ann Arbor, MI 48109-2143, USA. (ganuna@umich.edu)

(C)2013. American Geophysical Union. All Rights Reserved. 2169-9380/13/10.1002/jgra.50137
}

of field lines with the International Geomagnetic Reference Field (IGRF) 1980 as the internal field and the Tsyganenko and Usmanov [1982] model for the external field. They reported the 30 year secular variation as well as the seasonal and daily variation of the conjugate point. During solstices, the daily variation is largest (about $2^{\circ}$ in latitude and about $9^{\circ}$ in longitude for $K p=1$ ). Furthermore, the higher the magnetic activity, the larger the daily variation becomes. They concluded that more advanced magnetic mapping is required to solve the dislocation of the conjugate points during high geomagnetic activity.

[4] Conjugacy of large-scale auroral activity has been studied using simultaneous global satellite images of the auroral zone, namely, data from Visible Imaging System (VIS) Earth camera [Frank et al., 1995] on board the Polar satellite and images from Wideband Imaging Camera (WIC) on board the IMAGE satellite [Mende et al., 2000]. The two imaging spacecrafts were operating simultaneously during 2001 and 2002. The number of events with good viewing conditions for both hemispheres and interesting auroral activity occurring is limited, but nonetheless, some valuable science results have been reported based on this unique data set. For instance, interhemispheric comparison of the polar cap boundary demonstrated that the amount of open magnetic flux is equal between the hemispheres [Laundal et al., 2010]. Asymmetries in the polar cap boundary location up to $5^{\circ}$ in latitude were observed, 
and they arise when the magnetic flux closure rate is highest and in particular when the oval is contracting and the magnetotail flux is rapidly closing during sub-storm expansions. These inter-hemispheric asymmetries were explained by seasonal differences as the summer hemisphere responds to the magnetospheric convection changes faster than the winter hemisphere does.

[5] Simultaneous observations of 15 sub-storms demonstrated that the main contribution to the displacement of the auroral sub-storm location on the two hemispheres comes from the orientation of the interplanetary magnetic field (IMF), as the dawn-dusk component penetrates to the magnetosphere-ionosphere system [Østgaard et al., 2005]. A minor effect is controlled by the dipole tilt angle of the Earth. These observations qualitatively agree with the magnetic field model results of interhemispheric asymmetry as produced by the Tsyganenko T96 and T02 models [Tsyganenko, 1995, 2002a, 2002b]. The models predict the observed linear relationship between the azimuthal displacement of the sub-storms and the IMF clock angle or the dipole tilt angle but tend to underestimate the effect by an order of magnitude.

[6] A recent study by Saita et al. [2011] reported an attempt to study the interhemispheric displacement of substorms with a numerical magnetohydrodynamics (MHD) simulation of the solar wind-magnetosphere-ionosphere system. The simulation models by Tanaka et al. [2010] have been able to reproduce magnetospheric sub-storm signatures, such as formation of the near-Earth neutral line (NENL), tailward plasmoid release, and Earthward flows, as a consequence of the southward turning of the IMF. The magnetic field lines traced to the two hemispheres from the near-Earth magnetotail show approximately the displacements described by previous studies ( $4-5 \mathrm{~h}$ in magnetic local time (MLT)), even though the dipole tilt angle was set to zero for the simulation runs. Furthermore, the displacement during negative IMF $B_{y}$ was shown to be larger than the displacement during positive IMF $B_{y}$. Temporal evolution was also observed as the inter-hemispheric displacement gradually increased until the sub-storm onset. Asymmetric field line distortions on different hemispheres for constant IMF $B_{y}$ and non-tilted dipole axis was concluded to be mainly caused by the field-aligned current (FAC) distribution along the field lines in the high-pressure region of near-Earth space.

[7] Quite opposite to these studies that demonstrate the conjugacy of the aurora and IMF-controlled displacement of auroral activations, Laundal and Østgaard [2009] reported non-conjugate large-scale enhancements in the auroral precipitation pattern. Simultaneous global images of the two hemispheres showed strongly non-conjugate auroral intensifications with different intensities and different temporal evolution and almost opposite locations within the auroral oval during strongly positive IMF $B_{x}$. This peculiar observation is concluded to result from a predicted but previously unobserved inter-hemispheric FAC system, which originates in the region of high conductivity gradients near the terminators. Vorobjev et al. [2001] analyzed the coordinated Antarctic ground and Polar Ultraviolet observations to study aurora conjugacy during substorms. They found that the displacements of the poleward edge of the auroral bulge can be significant, up to $5^{\circ}$.
The sense and magnitude of displacements are related to the IMF orientations.

[8] For mesoscale auroral conjugate studies, the most commonly investigated conjugate stations are Japanese Syowa-Iceland station pairs [Sato et al., 2005; Motoba et al., 2010, 2012]. The conjugate point distance between the Icelandic Tjörnes station and the Antarctic Syowa station is of the order of a few tens of meters according to the IGRF model. The two stations have similar all-sky TV cameras, which allow visual tracing of similar auroral forms and their evolution to deduce the motion of the geomagnetic conjugate point throughout the period of auroral activity [Sato et al., 2005]. It was concluded that the northern hemispheric conjugate point of Syowa station moved about $200 \mathrm{~km}$ in longitude and about $50 \mathrm{~km}$ in latitude in an hour during steady solar wind and small IMF $B_{y}$ component. The studied event took place close to the equinox in September. Deformation of the magnetic field topology or a localized FAC was suggested to explain the observed conjugate point dislocation. However, it was clearly due to neither IMF $B_{y}$ polarity nor seasonal differences in ionospheric conditions between the hemispheres.

[9] The conjugate auroral sub-storm studied by Motoba et al. [2010] demonstrated well how rare fully conjugate auroral events are. Previous and following activations were reported to be completely displaced and dissimilar within the all-sky field of view (about $1000 \mathrm{~km}$ across at the auroral altitudes), but one weak sub-storm event in between showed strikingly similar and simultaneous structures. The maximum visually determined displacement was $3^{\circ}$ in latitude during the sub-storm expansion, while the MLT displacement (up to about $1 \mathrm{~h}$ ) varied faster in time but consistently with the IMF clock angle changes.

[10] In this paper, we investigate the effect of the dipole tilt angle and different solar wind and IMF parameters on the magnetic mapping between the hemispheres. As demonstrated by the previous studies, systematic analysis is needed to improve our understanding of the changing mapping properties. The range of different driving conditions during the limited number of conjugate point observations makes this a challenging task from an observational point of view. Thus, we use magnetic field modeling to provide an unlimited range and number of combinations of the tilt angle and solar wind parameters, but also, we analyze the previously reported conjugate events in more detail.

\section{Modeling Approach}

[11] To investigate the question of magnetic conjugacy, we follow the magnetic field lines from the northern to the southern hemisphere using the T02 Tsyganenko magnetospheric magnetic field model [Tsyganenko, 2002a, 2002b]. In the T02 model, the general approach is to parametrize the current systems and evaluate these parameter values in a statistical sense, using a large magnetospheric database. Several revisions were introduced in the mathematical description of the major sources of the magnetospheric field and in their parametrization with respect to the earlier T96 model [Tsyganenko, 1995]. A partial ring current with fieldaligned current closure is included, and the cross-tail current sheet is warped in two dimensions in response to the geodipole tilt, with its inner edge shifting along the Sun-Earth 
line and its thickness varying along and across the tail. The magnetopause is specified according to the empirical model by Shue et al. [1997]. The model parameters are geo-dipole tilt angle, IMF $B_{y}$ and $B_{z}$ components, solar wind dynamic pressure, and the Dst index. An attempt is made to take into account the preceding history of the solar wind by introducing two functions, G1 and G2, that depend on the IMF $B_{z}$ and solar wind velocity and their time history.

[12] We have chosen to use the T02 model from among the three most recent and available Tsyganenko models (such as T96, T02, and TS05) [Tsyganenko and Sitnov, 2005] due to several reasons. As compared to T96 model, (1) the T02 model contains the partial ring current and thus dawn-dusk asymmetry of the inner magnetosphere magnetic field and (2) the model has a better representation of the interconnection of IMF $B_{y}$ with the magnetospheric magnetic field. Simple dependencies on solar wind and IMF parameters are introduced into the model as two additional G1 and G2 input parameters. A later version, TS05, has a similar mathematical structure, but the data used were only for 37 storms with Dst $<-65 \mathrm{nT}$ that occurred between October 1996 and November 2000 [Tsyganenko et al., 2003]. In addition to Dst, solar wind dynamic pressure, and IMF $B_{y}$ and $B_{z}$, the TS05 model parameters include six variables $W_{i}, i=1,6$. These variables, $\mathrm{W}$, enter in the six magnitude coefficients for the magnetic fields from each source.

[13] In addition to the standard input parameters in the T02 model, we introduced the possibility to incorporate changes in the model magnetic field due to rotation of a tail current sheet from the Sun-Earth line. In most models, the tail current sheet is aligned with $X_{\mathrm{GSM}}$ line. In reality, it is not necessarily true, but the non-radial flow of the solar wind can cause the rotation of the tail current sheet [Hones et al., 1986; Tsyganenko et al. 1998]. When solar wind flow is radial with $V_{x}$ component dominant over small $V_{y}$ and $V_{z}$ components, the tail is aligned with the solar wind flow. When either of the $V_{y}$ and $V_{z}$ components are not close to zero, even small deviations of the tail location relative to the Sun-Earth line can cause notable variations in the magnetic field. In this study, we consider only a nonzero $V_{z}$ component. To take into account the rotation, we simply rotate the original GSM coordinate system in the $X Z_{\mathrm{GSM}}$ plane for a specific angle $\alpha$, which can be obtained from the solar wind flow data as $\tan \alpha=\frac{V_{z}}{V_{x}}$. This angle is the angle at which solar wind flows relative to the $X_{\mathrm{GSM}}$ axis. Then we compute the external magnetic field from the T02 model in this coordinate system, where the tail current sheet is again directed along the $X_{\mathrm{GSM}}$ axis. In fact, the rotation will result in a change of an effective "tilt angle," which is defined as an angle between the $Z_{\mathrm{GSM}}$ axis direction and the dipole axis direction. From that point of view, changes coming from taking into account the real direction of the solar wind flow are the same as the changes coming from dipole angle change, and depending on the direction of the solar wind may both increase or decrease the "effective dipole tilt."

\section{Differences in Latitudinal Conjugacy in the Northern and Southern Hemispheres}

[14] We start from the circles of latitudes from $55^{\circ}$ to $75^{\circ}$ with $5^{\circ}$ step at all MLTs with 15 min step in the northern hemisphere. We trace magnetic field lines from these points in the northern hemisphere to the corresponding points in the southern hemisphere using the T02 Tsyganenko magnetic field model. We use the corrected geomagnetic coordinate (CGM) system to remove the non-dipolar effects from the internal magnetic field. CGM coordinates of a point are computed by tracing the magnetic field line using internal field model (IGRF in our case) through the specified point to the dipole geomagnetic equator and then returning to the same altitude along the dipole field line and assigning the obtained dipole latitude and longitude as the CGM coordinates to the starting point. Using of the corrected coordinates results in the geometrically symmetrical northern grid seen in Figure 1. Figure 1 presents the results of this mapping by red lines in the northern hemisphere and blue lines in the southern hemisphere. The mapping was done for three dipole tilt angles, namely, for northern winter $-33^{\circ}$ (winter) shown on the left-side plots, $0^{\circ}$ (equinox) in the middle, and for northern summer $+33^{\circ}$ (summer) on the right-side plots. The upper row of polar plots in Figure 1 was computed for a quiet period with $P_{s w}=2 \mathrm{nPa}$, Dst $=10 \mathrm{nT}$, IMF $B_{y}=0 \mathrm{nT}$, and IMF $B_{z}=10 \mathrm{nT}$ as input parameters to the T02 model (Figures 1a-1c). The row of plots in the middle corresponds to the moderately disturbed conditions with $P_{s w}=2 \mathrm{nPa}$, Dst $=-20 \mathrm{nT}$, IMF $B_{y}=0 \mathrm{nT}$, and IMF $B_{z}=-5 \mathrm{nT}$ (Figures 1d-1f). The bottom row presents the results for disturbed conditions reflected in the T02 parameters, such as $P_{s w}=8 \mathrm{nPa}$, Dst $=-100 \mathrm{nT}$, IMF $B_{y}=0 \mathrm{nT}$, and IMF $B_{z}=-20 \mathrm{nT}$ (Figures 1g-1i).

[15] As can be seen, for the quiet period, the deviations in latitudes for magnetic field lines coming from the northern hemisphere (red circles) to the southern hemisphere (blue circles and lines) is present only for higher latitudes $\left(75^{\circ}\right)$ and for dipole tile angles corresponding to winter (Figure 1a) and summer (Figure 1c). For zero dipole tilt angle (Figure 1b), the magnetic field lines starting from the northern hemisphere at circles with latitudes $55^{\circ}$ to $75^{\circ}$ come to the same circles of latitudes in the southern hemisphere. It should be noted that here we can detect only the differences in latitudes for magnetic field lines connecting both hemispheres, not in longitudes.

[16] For moderately disturbed conditions during equinox (Figure 1e), magnetic field lines have their both ends at the same latitudes from $55^{\circ}$ to $70^{\circ}$ in the northern and southern hemispheres. At the same time, the magnetic field lines starting at $75^{\circ}$ latitudes on the dayside in the northern hemisphere are open in the 08-16 MLT sector in terms of that they do not come to the southern hemisphere. Magnetic field lines starting at $75^{\circ}$ latitudes do not come to the southern hemisphere on the dayside in the 07-17 MLT sector for dipole tilt angle $-33^{\circ}$ (Figure 1d), and they are open both on the dayside (10-14 MLT) and nightside (21-03 MLT) for dipole tilt angle $+33^{\circ}$ (Figure 1f). The deviations in latitudes can be seen for magnetic field lines starting at $70^{\circ}$ in the northern hemisphere.

[17] For the disturbed period, only magnetic field lines starting at $55^{\circ}$ in the northern hemisphere come to the same latitudes to the southern hemisphere during winter (Figure 1g) and summer (Figure 1i) times. For winter time, the magnetic field lines starting at $65^{\circ}$ and $70^{\circ}$ are open on the dayside (07-17 MLT) and field lines starting at $75^{\circ}$ come all the way to the southern hemisphere only in the 
Dipole tilt angle $=-33^{\circ}$, winter Dipole tilt angle $\sim 0^{\circ}$, equinox Dipole tilt angle $=+33^{\circ}$, summer

Quiet period: $\mathrm{Psw}=2 \mathrm{nPa}$, Dst $=10 \mathrm{nT}$, IMF By $=0 \mathrm{nT}, \mathrm{IMF} \mathrm{Bz}=10 \mathrm{nT}$
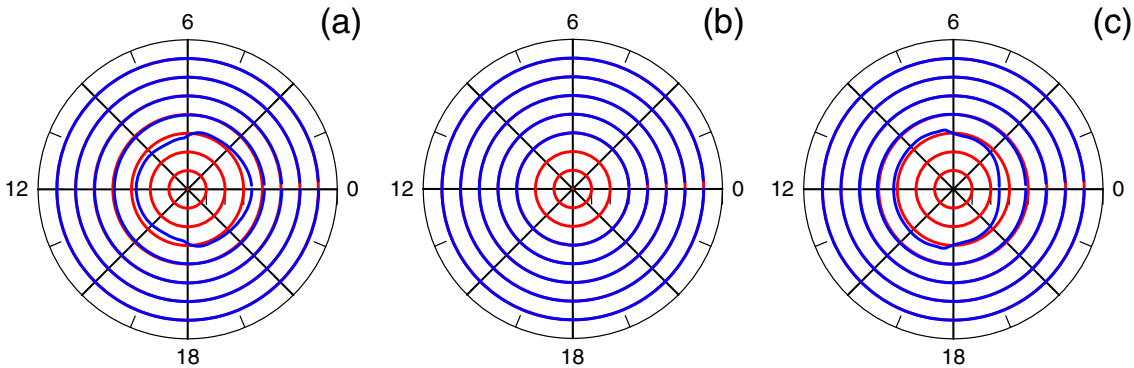

(c)

Moderately disturbed period: $\mathrm{Psw}=2 \mathrm{nPa}, \mathrm{Dst}=-20 \mathrm{nT}, \mathrm{IMF} B y=0 \mathrm{nT}, \mathrm{IMF} B z=-5 \mathrm{nT}$
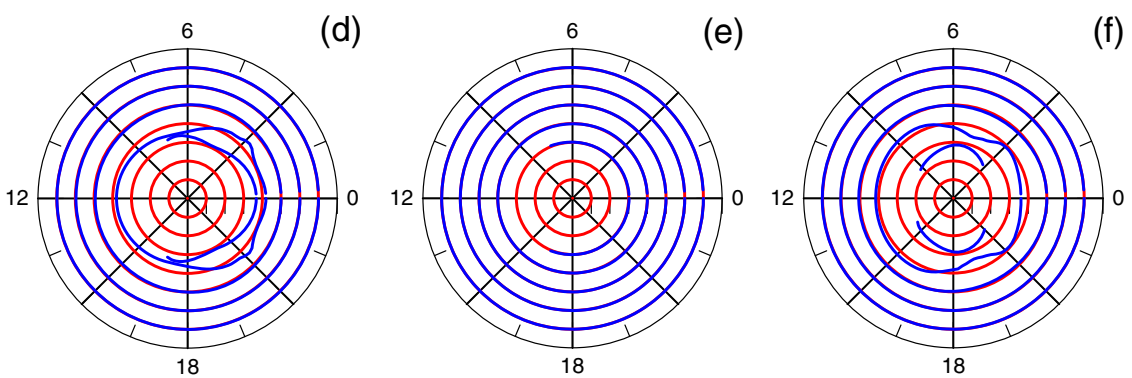

(f)

Disturbed period: Psw $=8 \mathrm{nPa}$, Dst $=-100 \mathrm{nT}$, IMF By $=0 \mathrm{nT}, \mathrm{IMF} B z=-20 \mathrm{nT}$
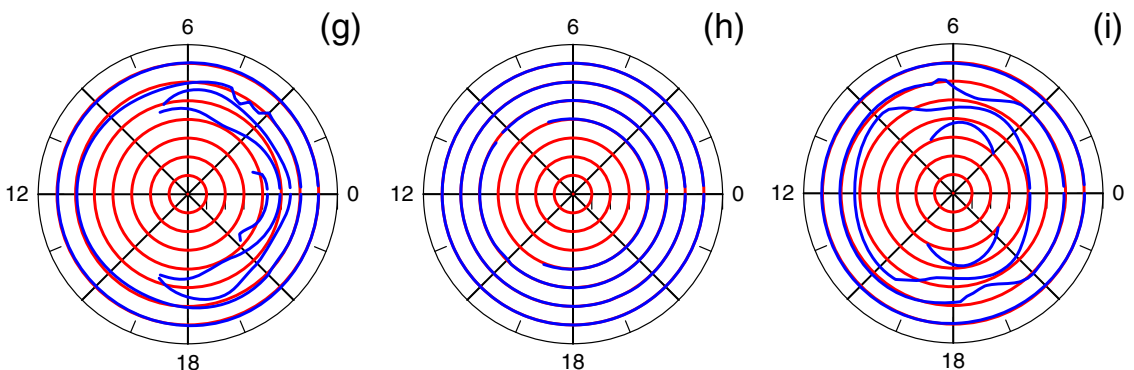

Figure 1. Latitudinal positions of field lines in the northern (red) and southern (blue) hemispheres showing latitudinal conjugacy.

narrow 22-03 MLT sector around midnight. For summer time the magnetic field lines starting at $70^{\circ}$ are open on the dayside (08-16 MLT) and on the nightside (21-02 MLT). All magnetic field lines starting at $75^{\circ}$ do not come to the southern hemisphere. For zero tilt angle (Figure $1 \mathrm{~h}$ ), magnetic field lines are closed and at the same latitudes in both hemispheres for $55^{\circ}, 60^{\circ}$, and $65^{\circ}$. Field lines are open on the dayside in 08-16 MLT sector for $70^{\circ}$ and at all MLTs for $75^{\circ}$.

[18] The latitudinal differences exhibit the reversed asymmetry depending on the season. The magnetic field lines come to southern hemisphere at higher latitudes on the dayside and at lower latitudes on the nightside in winter (Figures 1a, 1d, and 1g) and vice versa in summer (Figures 1c, 1f, and 1i). For summer, the magnetosphere is more open (field lines starting at $75^{\circ}$ in northern hemisphere do not come to the southern hemisphere at all).

\section{Parameters Influencing the Interhemispheric Magnetic Conjugacy}

[19] The magnetic conjugacy depends on many factors including the solar wind and IMF parameters, dipole tilt angle, and conditions in the magnetosphere and its configuration. Since we use the T02 model to follow the magnetic field lines, we can study the latitudinal and longitudinal dependencies of conjugate points on the model parameters, such as solar wind dynamic pressure $P_{s w}$ and IMF $B_{y}$ and $B_{z}$. Dependence on dipole tilt angle can be revealed by tracing magnetic field lines on different months during the year. Simple rotation of the original GSM coordinate system in $X Z_{\mathrm{GSM}}$ plane for a specific angle $\alpha$, obtained as $\tan \alpha=\frac{V_{z}}{V_{x}}$, also allow us to investigate the influence of rotation of the tail current sheet from the Sun-Earth line. By doing this, we can study the influence of non-radial solar wind flow on the magnetic field configuration, magnitude, and magnetic conjugacy. The above-mentioned dependencies are studied by following the magnetic field lines, which start at the circle of $70^{\circ}$ at all MLTs in the northern hemisphere, to the southern hemisphere. According to Figure 1, the moderately disturbed period suits best for this study, since the magnetic field lines starting at $70^{\circ}$ latitudes exhibit latitudinal differences in the southern hemisphere and are not open for both winter and summer times. The quiet period mapping lacks significant latitudinal deviations, and the magnetic field lines 
(a)

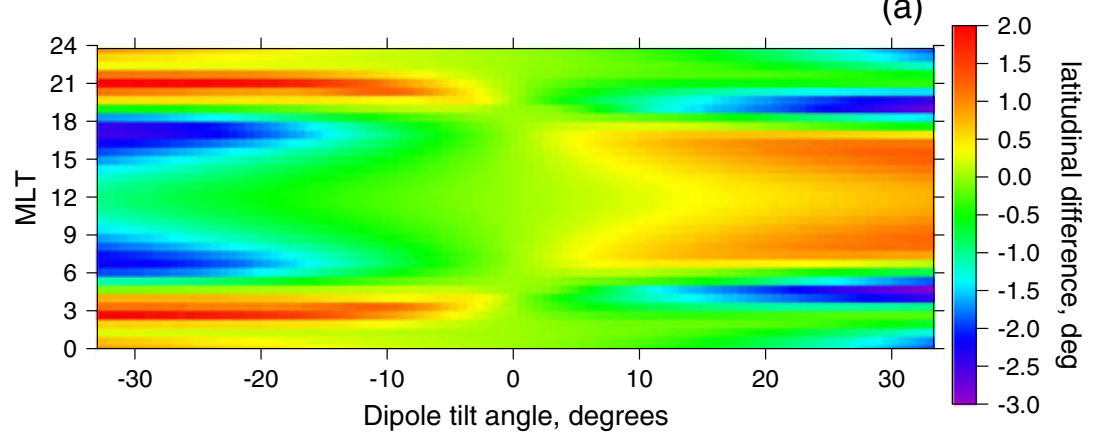

(b)

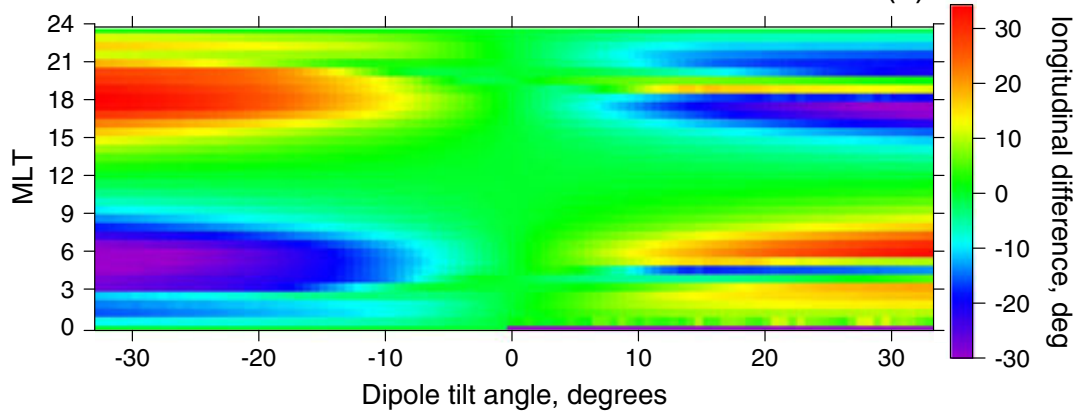

Figure 2. Differences in latitudinal and longitudinal conjugacy as dependent on the dipole tilt angle.

are already open for $65^{\circ}$ of latitude for the disturbed period. We use the corrected geomagnetic coordinate system as was mentioned in the previous section.

\subsection{Dipole Tilt Angle}

[20] To produce Figure 2, the magnetic field lines were followed from the circle of $70^{\circ}$ at all MLTs with $15 \mathrm{~min}$ step from the northern hemisphere to the southern hemisphere for dipole tilt angles from $-33^{\circ}$ to $+33^{\circ}$ with $3^{\circ}$ step during moderately disturbed conditions $\left(P_{s w}=2 \mathrm{nPa}\right.$, Dst $=-20 \mathrm{nT}$, IMF $B_{y}=0 \mathrm{nT}$, IMF $B_{z}=-5 \mathrm{nT}$ ). Figure $2 \mathrm{a}$ presents the latitudinal difference $\Delta \lambda=\lambda_{N}-\lambda_{S}$ between the starting latitude $\lambda_{N}$ of a magnetic field line in the northern hemisphere (set to $70^{\circ}$ ) and the final latitude $\lambda_{S}$ in the southern hemisphere. Positive values mean that the magnetic field comes to the southern hemisphere at lower latitudes than it started in the northern hemisphere, and negative values at higher latitudes. Figure $2 \mathrm{~b}$ shows the longitudinal differences $\triangle M L T=M L T_{N}-M L T_{S}$ in degrees between the starting $M L T_{N}$ of a magnetic field line in the northern hemisphere and the final $M L T_{S}$ in the southern hemisphere. Positive values correspond to magnetic field lines coming to the southern hemisphere earlier in MLT compared to the MLT they started from in the northern hemisphere, and negative values to later MLTs.

[21] As can be seen in Figure 2a, the dependence of the latitudinal difference on the tilt angle and MLT is symmetric relative to noon being very similar on dawn and dusk MLTs. The latitudinal difference can reach values of several degrees. During equinox, there is no difference for any MLT. For large negative tilt angles, the difference is $-2.5^{\circ}$ at 06-08 MLT and 16-18 MLT and persist around these MLTs with increasing tilt angle up to about $-10^{\circ}$. The difference of $+2^{\circ}$ exists at 02-04 MLT and 20-22 MLT and is present for tilt angles up to $-5^{\circ}$. For positive tilt angles, the picture is reversed with $+2^{\circ}$ at 06-08 MLT and 16-18 MLT and $-2.5^{\circ}$ at 02-04 MLT and 20-22 MLT.

[22] At the same time, the longitudinal differences can reach values of several tens of degrees (Figure 2a), and the symmetry is reversed related to noon. For negative tilt angles, the differences are from $-30^{\circ}$ to $-5^{\circ}$ at around $04-08$ MLT and from $+30^{\circ}$ to $+5^{\circ}$ at around 16-20 MLT. For positive tilt angles, the pattern is reversed with an appearance of narrow bands of longitudinal differences with opposite signs as $+15^{\circ}$ at 19 MLT and $-15^{\circ}$ at 05 MLT. This is due to the complex configuration of model magnetic field lines at dawn and dusk.

\subsection{Solar Wind Dynamic Pressure}

[23] To study the dependence of magnetic conjugacy on the solar wind dynamic pressure (Figure 3), the magnetic field lines were followed from the circle of $70^{\circ}$ at all MLTs with 15 min step from the northern hemisphere to the southern hemisphere for dipole tilt angles of $-33^{\circ}$ and $+33^{\circ}$ during moderately disturbed conditions (Dst $=-20 \mathrm{nT}$, IMF $B_{y}=$ $0 \mathrm{nT}$, IMF $B_{z}=-5 \mathrm{nT}$ ) and varying solar wind dynamics pressure values from $1 \mathrm{nPa}$ to $20 \mathrm{nPa}$ with $1 \mathrm{nPa}$ step. The latitudinal difference (Figures $3 \mathrm{a}$ and $3 \mathrm{~b}$ ) can be as large as $2^{\circ}$ and longitudinal difference as large as $20^{\circ}$ (Figures $3 \mathrm{c}$ and $3 \mathrm{~d}$ ), and the picture is similar but reversed in sign in summer compared to winter. For $P_{s w}$ below $10 \mathrm{nPa}$, the latitude differences vary no more than $\pm 1^{\circ}$ for both winter (Figure 3a) and summer (Figures $3 b$ ) times for all MLTs. Maximum latitudinal difference of $\pm 2^{\circ}$ is present only around midnight for large values of $P_{s w}>10 \mathrm{nPa}$. This day-night asymmetry in the latitudinal difference is observed for both winter and summer. The significant longitudinal difference of $\pm 15-20^{\circ}$ appears for $P_{s w}>12 \mathrm{nPa}$ on dawn and dusk for both winter (Figure 3c) and summer (Figure 3d) times. Positive difference is at 16-22 MLT (02-08 MLT) for winter (summer) and negative is at 02-08 MLT (16-22 MLT) for winter (summer). 

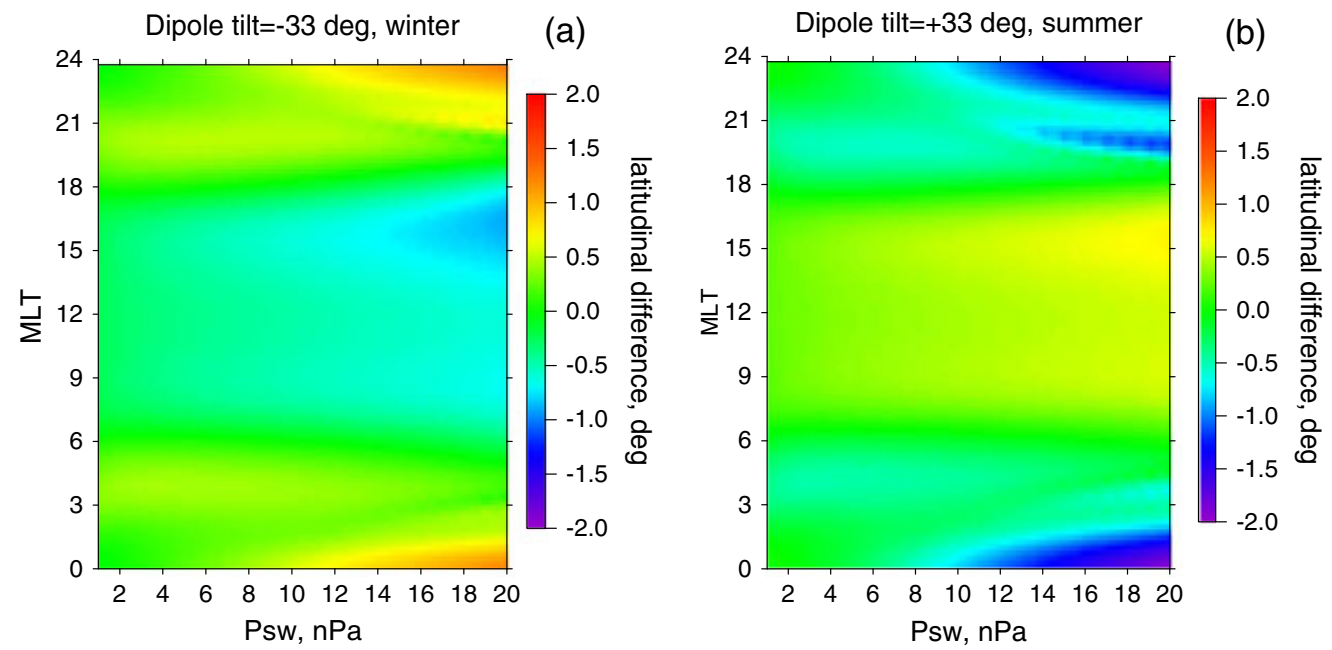

(c)
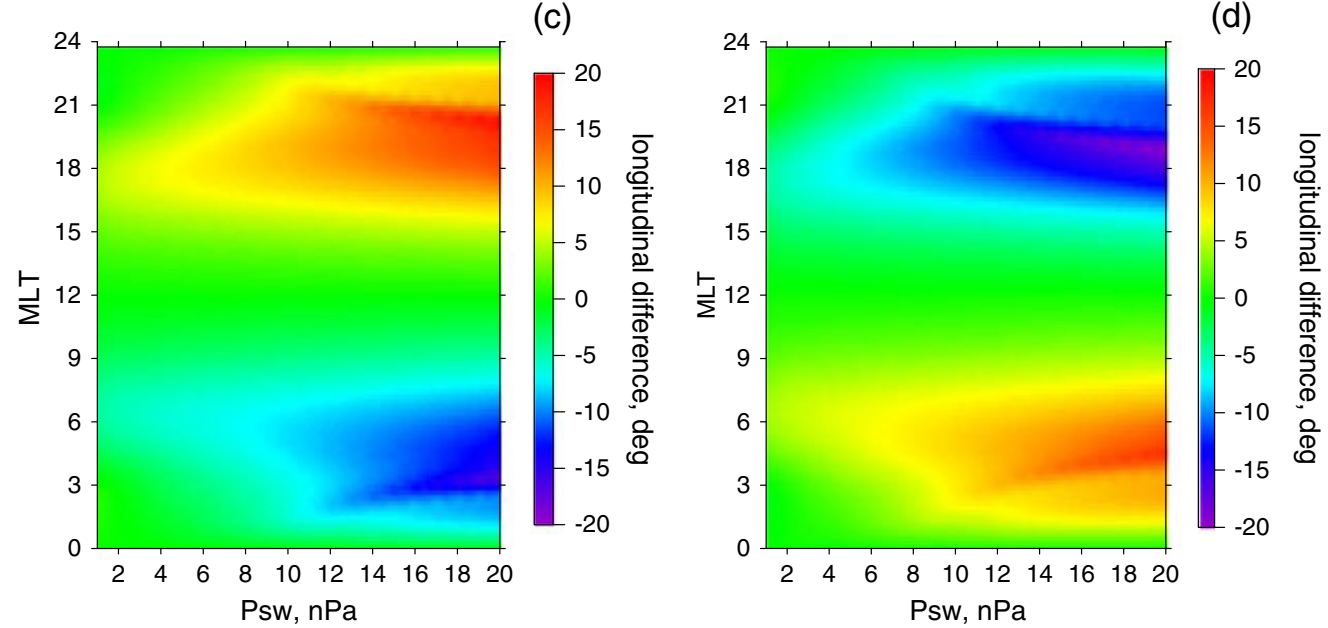

Figure 3. Magnetic conjugacy dependence on solar wind dynamic pressure.

There is no dependence on $P_{s w}$ magnitude on the dayside (09-15 MLT), but, even though the longitudinal difference was close to zero around midnight, already at 22 and 02 MLT, it was $10^{\circ}$, so the day-night asymmetry is also present for longitudinal difference. In general, conjugacy in the nightside magnetosphere is much more affected by large solar wind dynamic pressure.

\subsection{IMF $B_{\mathrm{y}}$}

[24] Since the T02 model contains IMF $B_{y}$ as an input parameter, we varied it from $-18 \mathrm{nT}$ to $+18 \mathrm{nT}$ and mapped the magnetic field lines from a $70^{\circ}$ latitude circle in the northern hemisphere to the southern hemisphere (Figure 4) during moderately disturbed conditions $\left(P_{s w}=2 \mathrm{nPa}\right.$, Dst $=-20 \mathrm{nT}$, IMF $\left.B_{z}=-5 \mathrm{nT}\right)$. Similar to the dependence on solar wind dynamic pressure, the pictures in Figure 4 for northern winter and summer are reversed in terms of the sign of latitudinal and longitudinal differences and also in terms of the sign of IMF $B_{y}$. The obtained latitudinal difference is quite small, within $\pm 1^{\circ}$ for both winter (Figure $4 \mathrm{a}$ ) and summer (Figure $4 \mathrm{~b}$ ) times. It reaches $-2^{\circ}$ only for $B_{y}>15 \mathrm{nT}$ at 03-06 MLT and $B_{y}<-15 \mathrm{nT}$ at 18-21 MLT in summer. Negative $15-20^{\circ}$ of longitudinal difference is present also only for $B_{y}>15 \mathrm{nT}$ at 21-06 MLT for winter and 18-03
MLT for summer with small MLT area of 15-19 MLT for $B_{y}<-15 \mathrm{nT}$ in summer. Positive $15-20^{\circ}$ of longitudinal difference exists also only for $B_{y}<-15 \mathrm{nT}$ at $18-03$ for winter and at 21-06 MLT for summer with small MLT area of 04-09 MLT for $B_{y}>15 \mathrm{nT}$ in summer. For other IMF $B_{y}$, the difference does not exceed about $\pm 5^{\circ}$. The asymmetry of conjugate points is present due to nonzero values of IMF $B_{y}$, but there is only a weak dependence on the magnitude of IMF $B_{y}$. The asymmetry depends on MLT and dipole tilt angle.

\subsection{IMF $B_{z}$}

[25] We varied IMF $B_{z}$ from $-20 \mathrm{nT}$ to $+20 \mathrm{nT}$ as an input parameter to the T02 model and mapped the magnetic field lines from $70^{\circ}$ latitude circle in the northern hemisphere to southern hemisphere (Figure 5) during moderately disturbed conditions $\left(P_{s w}=2 \mathrm{nPa}\right.$, Dst $=-20 \mathrm{nT}$, IMF $\left.B_{y}=0 \mathrm{nT}\right)$ for dipole tilt angles of $-33^{\circ}$ and $+33^{\circ}$. The white areas seen in Figures $5 \mathrm{a}$ and $5 \mathrm{c}$ for large negative IMF $B_{z}$ correspond to the magnetic field lines which are open and do not have their foot points in the southern hemisphere when leaving the northern hemisphere. The influence of IMF $B_{z}$ on interhemispheric conjugacy depends very much on the sign of IMF $B_{z}$ but not much on the magnitude. As can be seen in 

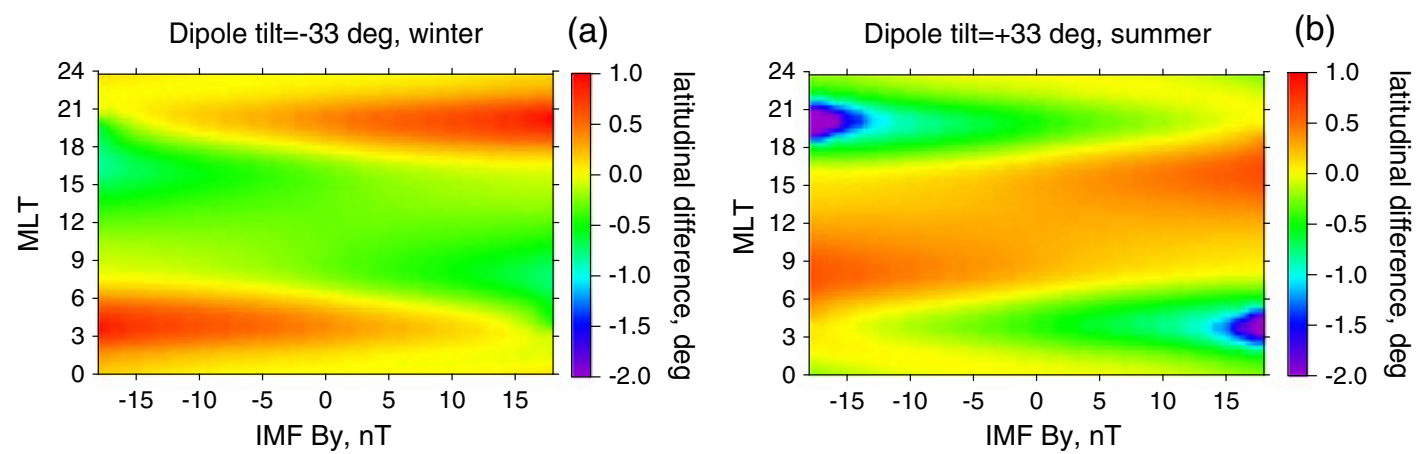

(c)

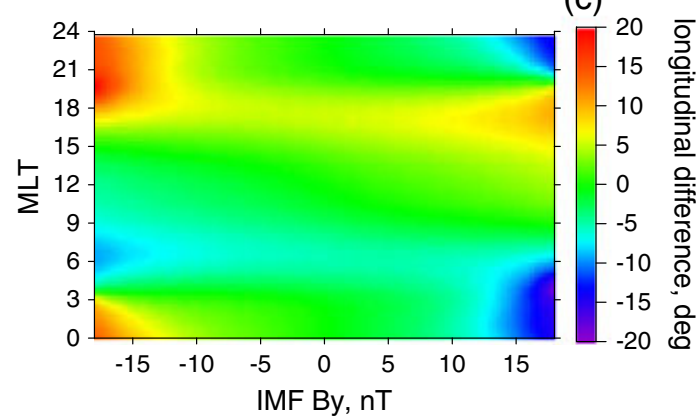

(d)

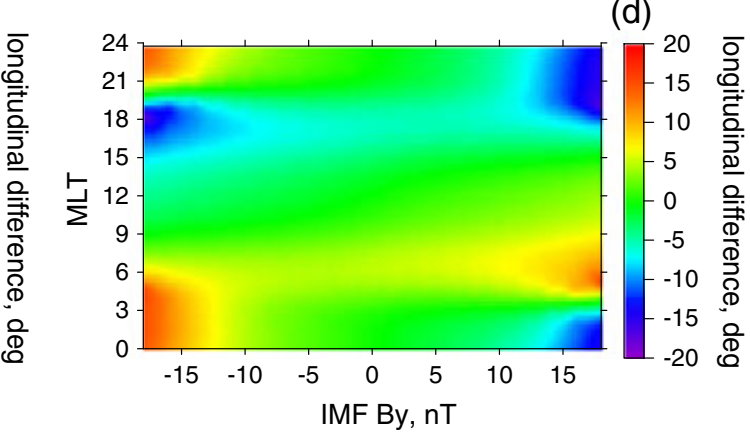

Figure 4. Magnetic conjugacy dependence on IMF $B_{y}$.
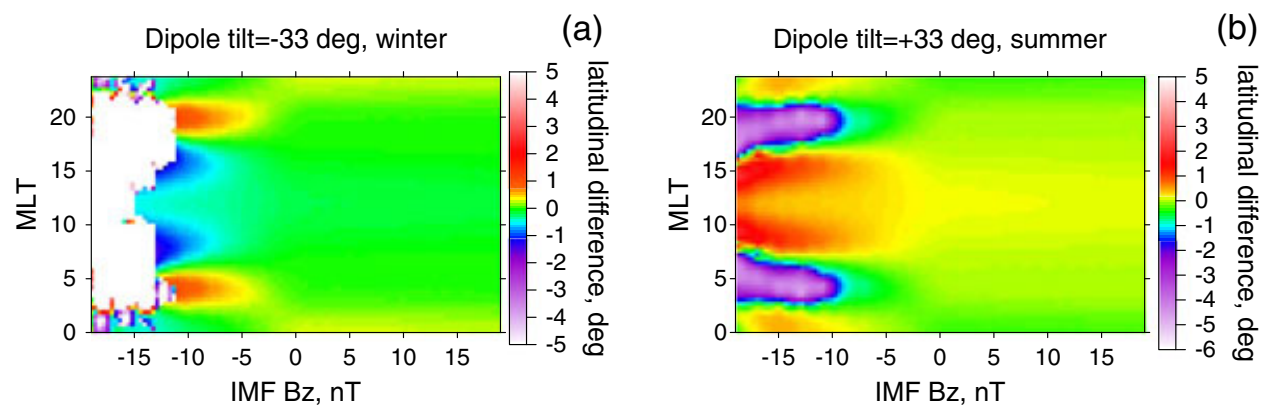

(c)

(d)
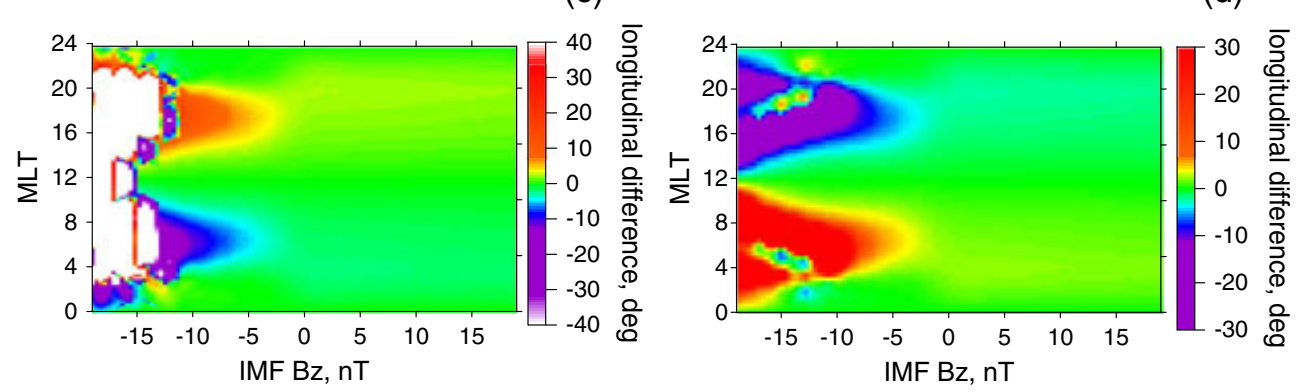

Figure 5. Magnetic conjugacy dependence on IMF $B_{z}$.

Figures $5 \mathrm{a}$ and $5 \mathrm{~b}$, the latitudinal difference is close to zero for positive IMF $B_{z}$ in northern winter and summer. The picture is very different for negative IMF $B_{z}$. The latitudinal difference can reach 3 or $4^{\circ}$ in absolute values around 20 and 05 MLT. The longitudinal difference is not large, but it also shows a clear dependence on the sign of IMF $B_{z}$, not much on the magnitude. For positive IMF $B_{z}$, the difference of about $+2^{\circ}$ is on the duskside and $-2^{\circ}$ on the dawnside (Figure 5c) in winter and reverse (Figure $5 \mathrm{~d}$ ) in summer.
It increases up to $10^{\circ}$ for negative IMF $B_{z}$ and becomes more localized at around 18 and 6 MLTs.

\subsection{Rotation of Neutral Sheet from the Sun-Earth Line}

[26] Figure 6 demonstrates the dependence of the latitudinal and longitudinal differences between the northern and southern hemispheric foot points on the rotation angle of the neutral sheet from the Sun-Earth line. Figure $6 \mathrm{a}$ shows the latitudinal differences for the magnetic field lines 
(a)

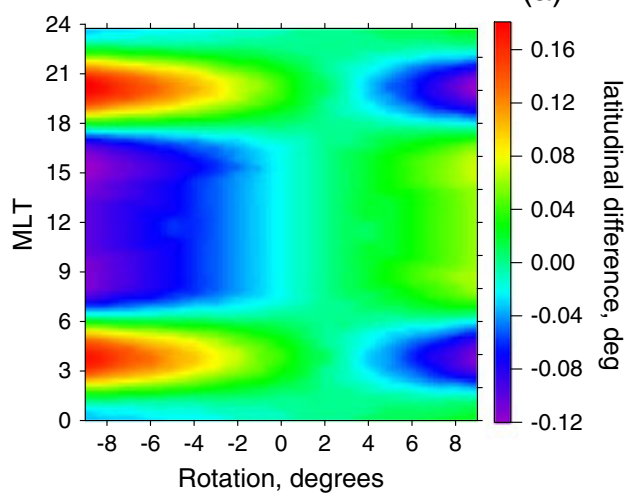

(c)

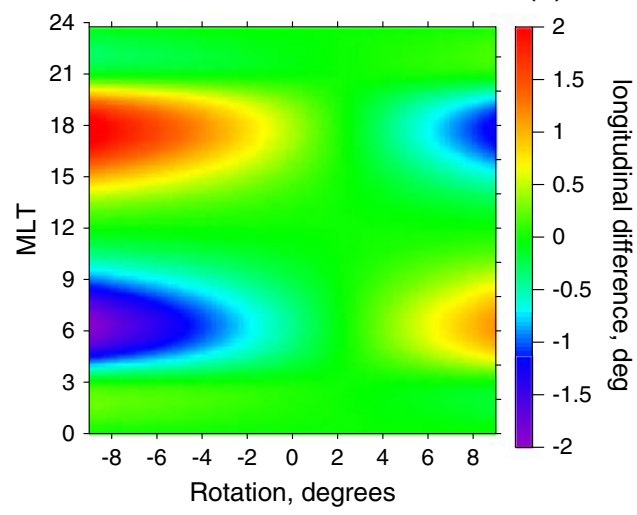

(b)

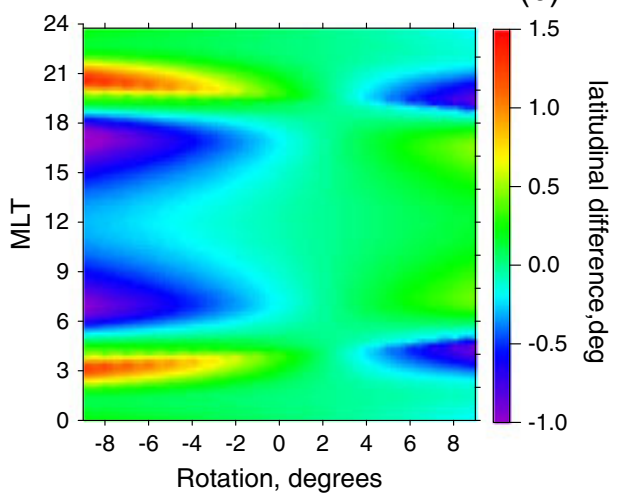

(d)

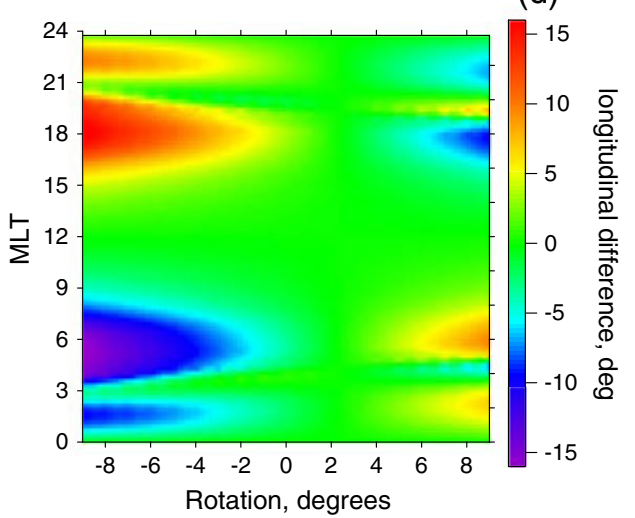

Figure 6. Dependence on rotation of neutral sheet from Sun-Earth line.

starting at a circle of $65^{\circ}$ latitude and Figure $6 \mathrm{~b}$ for the magnetic field lines starting at a circle of $70^{\circ}$ of latitude in the northern hemisphere for rotation angles from $-9^{\circ}$ to $9^{\circ}$. Negative values of rotation angles $\tan \alpha=\frac{V_{z}}{V_{x}}$ mean that the neutral sheet is above the Sun-Earth line, and positive values mean that it is below the Sun-Earth line.

[27] For the circle of $65^{\circ}$ latitude, the differences are very small, less than $0.2^{\circ}$. When the circle is moved poleward by $5^{\circ}$, the difference becomes as large as $-1.0^{\circ}$ to $1.5^{\circ}$. The patterns of the latitudinal difference dependence on the rotation angle and MLT are symmetric relative to the MLT $=12$ line for both starting latitudes. The largest positive differences are reached for negative rotation values $<-4^{\circ}$ at dawn and dusk for both starting latitudes. The largest negative differences are present at dawn and dusk for positive rotation angles $>4^{\circ}$ and at 06-18 MLT for negative rotation values $<-4^{\circ}$.

[28] The longitudinal difference is small (only $\pm 2^{\circ}$ ) for magnetic field lines starting at $65^{\circ}$ (Figure 6c). It reaches the maximum positive values at dusk (dawn) and the maximum negative values at dawn (dusk) for negative rotation angle values $<-4^{\circ}$ (positive rotation angle values $>7^{\circ}$ ), respectively. The picture is reversed relative to the MLT $=12$ line. For the higher starting latitude of $70^{\circ}$, the longitudinal differences are 7 times larger, being $\pm 15^{\circ}$ (Figure 6d). Similar structure of the locations of largest positive and negative differences is present as in Figure 6c with additional areas with similar signs of longitudinal differences closer to midnight.

\section{Conjugate Points in the Northern and Southern Hemispheres: Observed and Modeled}

[29] While there are plenty of ground-based magnetic field observations from both hemispheres (one recent example is by Viljanen and Tanskanen, [2012]), only a few simultaneous auroral observations made in the northern and southern hemispheres were done. One of them was reported by Sato et al. [2005] where two similar auroras were simultaneously acquired with all-sky TV cameras at the Tjörnes (Iceland) and Syowa (Antarctica) magnetically conjugate observatories. The magnetic latitude (MLAT) and longitude (MLON) of the two stations are $66.5^{\circ} \mathrm{N}$ and $73.1^{\circ} \mathrm{E}$ for Tjörnes and $66.1^{\circ} \mathrm{S}$ and $71.6^{\circ} \mathrm{E}$ for Syowa. Thus, their locations were ideal for studying geomagnetic conjugacy of auroras. The event occurred during magnetic pre-midnight at 2230-2400 UT on 26 September 2003. One of the results of this study was the existence of both latitudinal (poleward $\sim 50 \mathrm{~km}$ ) and longitudinal (westward $\sim 200 \mathrm{~km}$ ) displacement of the conjugate point in the southern hemisphere after $1 \mathrm{~h}$ of observations. Since the auroral images were so similar, it was possible to follow the conjugate points at both hemispheres and test the accuracy of the Tsyganenko models. Sato et al. [2005] found that none of the T96 and T02 Tsyganenko models used in this study could reproduce the observed displacement with the observed solar wind and IMF parameters, even taking into account the possible displacement due to the IMF $B_{y}$ component and the possible time delay. However, with the 


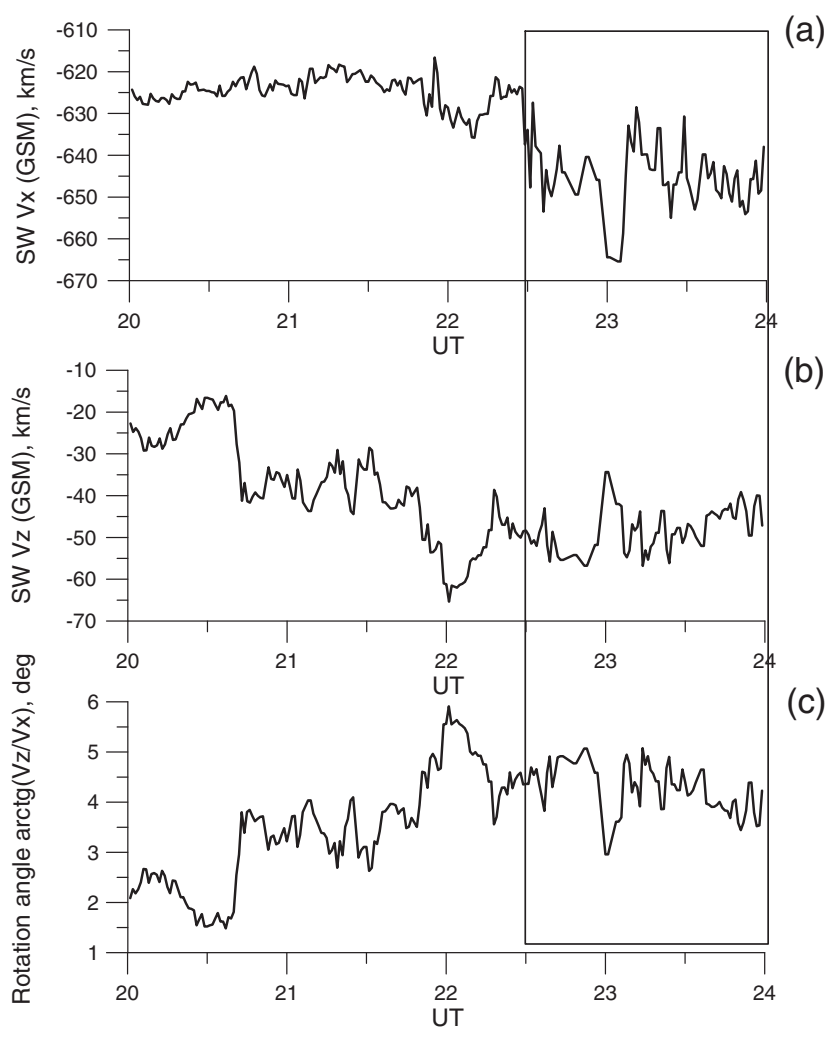

Figure 7. (a) $V_{x}$ and (b) $V_{z}$ components of the solar wind velocity observed during 20-24 UT on 26 September 2003 and (c) the computed rotation angle $\alpha=\operatorname{arctg}\left(\frac{V_{z}}{V_{x}}\right)$ of the tail current sheet from the Sun-Earth line. Black rectangle shows the time of the observed conjugate auroras in both hemispheres.

$V_{z}$ correction to the T02 model, we find that the locations of the conjugate aurora could be explained.

[30] To verify this result, we first examined the observed parameters of the solar wind during 20-24 UT on 26 September 2003 . We used the $5 \mathrm{~min}$ averaged data provided by OMNIWeb (http://omniweb.gsfc.nasa.gov/form/omni_min. html) and found that the period was characterized by a significant deviation of the solar wind from the radial (SunEarth) direction. Figure 7 presents (a) $V_{x}$ and (b) $V_{z}$ components of the observed solar wind velocity and (c) the computed rotation angle $\alpha=\arctan \left(\frac{V_{z}}{V_{x}}\right)$ of the tail current sheet from the Sun-Earth line. The black rectangle shows the time of the observed conjugate auroras in both hemispheres. It can be seen that during the marked period, the magnitude of $V_{z}$ was as large as $55 \mathrm{~km} / \mathrm{s}$, and the rotation angle can be as large as $3-5^{\circ}$. The magnetospheric plasma sheet is supposed to follow the solar wind flow direction [Tsyganenko and Fairfield, 2004], but the possible delays related to variable solar wind flow velocity are still unknown.

[31] Next, we analyzed the mapping changes, which may be caused by the inclination of the plasma sheet from the $X_{\mathrm{GSM}}$ axis. We mapped the location of the Tjörnes observatory in the northern hemisphere to the southern hemisphere and obtained the foot point of the corresponding magnetic field line there. We use the Tsyganenko T02 magnetic field model, in which the real direction of solar wind flow was taken into account by rotating the model neutral plasma sheet by the observed angle (Figure 7c). We used the 5 min averaged solar wind velocity values without any time delay. The result is given in Figure 8.

[32] Figure 8 presents the evolution of (a) the geographic latitude and (b) longitude of the foot points of the Tjörnes station in the southern hemisphere. The blue lines in both Figures $8 \mathrm{a}$ and $8 \mathrm{~b}$ show the latitude and longitude of the Tjörnes station obtained using the T02 model, which does not take into account non-radial solar wind flow. The black lines show the latitude and longitude of the Tjörnes station computed using the T02 model with the additional rotation angle procedure, described above. The red arrows indicate the displacement of the location of the conjugate aurora in latitude and longitude at around $2230 \mathrm{UT}$ during $1 \mathrm{~h}$ of observations.

[33] It can clearly be seen that the foot point of the Tjörnes station in the southern hemisphere moves poleward and westward during the period of observations, which corresponds to the observed conjugate point displacement. The meridional displacement is $\sim 0.5^{\circ}$ poleward, which corresponds to $\sim 50 \mathrm{~km}$, and the azimuthal shift is $\sim 1.6^{\circ}$ westward, which is about $\sim 80 \mathrm{~km}$. The value of the modeled poleward displacement is close to the observed one. Thus, we can conclude that the configurational changes of the magnetic field resulting from the variations of the solar wind and dipole tilt are one of the main reasons for the conjugate points displacements in latitude. It is important not only to take into account the influence of the IMF $B_{y}$ 

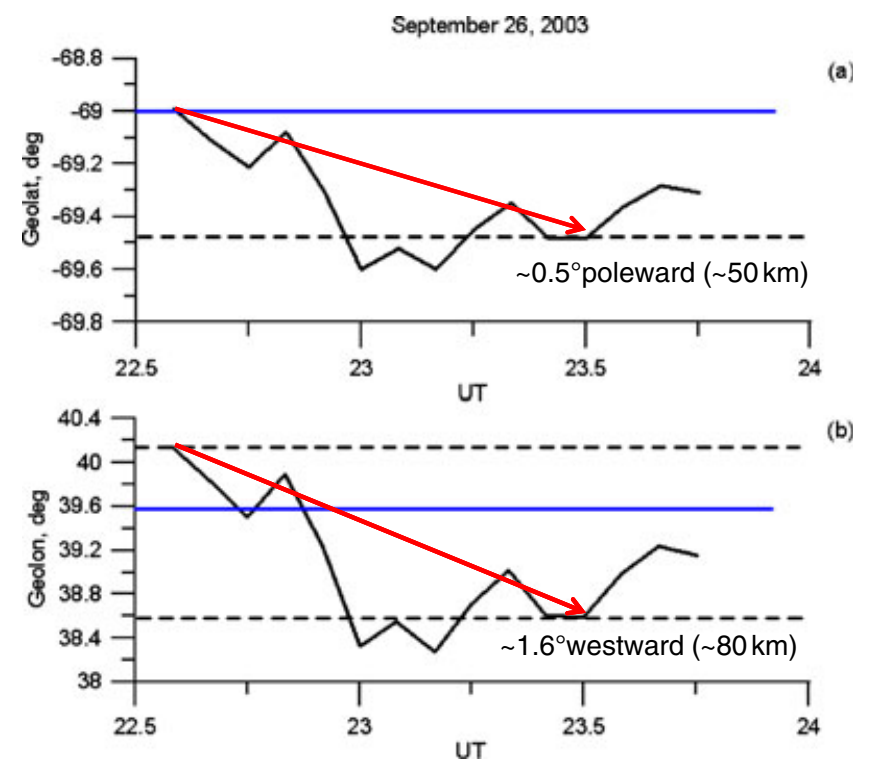

Figure 8. (a) The geographic latitude and (b) longitude of the foot points of the Tjörnes station in the southern hemisphere obtained by using the T02 model (blue lines) and using the T02 model with the additional rotation angle procedure (black lines). Red arrows indicate the displacement of the initial latitude and longitude at around 2230 UT during $1 \mathrm{~h}$ of observations [Sato et al., 2005].

component resulting in twisting of the magnetic field lines but also to consider the solar wind flow direction when it deviates from radial by more than $1-2^{\circ}$ (Figure $7 \mathrm{c}$ ). At the same time, the modeled longitudinal displacement is two times smaller than the observed.

\section{Discussion and Conclusions}

[34] The concept of the inter-hemispheric magnetic conjugacy was investigated by finding the conjugate points in the northern and southern hemispheres and analyzing their dependence on the dipole tilt angle, solar wind and IMF parameters, and conditions in the magnetosphere and its configuration. The Tsyganenko T02 magnetic field model was used to follow the magnetic field lines in the corrected geomagnetic coordinate system. Since observations of two points of magnetic field line are not possible, we can only use models. The better the model, the better the chance that we will obtain real conjugacy with the model. As it was stated in the description of our modeling approach, this model fits the best for our study from all the available models, since the earlier version T96 does not include the dawn-dusk asymmetry of the inner magnetosphere and the TS05 model was constructed only for storm times. The T02 model has its limitations, such as (1) it is valid only inside $15 R_{E}$ and (2) it does not include sub-storm variations of the magnetic field. The $15 R_{E}$ limitation is important for highly disturbed periods, when extreme currents form in the middle part of the plasma sheet. Due to them, negative $B_{z}$ could occur in the magnetotail. These highly disturbed periods are not the subject of the present study; we concentrate on moderately disturbed conditions. Similarly, substorm variations are beyond our consideration; we study the configurations, for which averaging over a sub-storm time should be valid.

[35] In our study, we set three levels of geomagnetic activity (quiet, moderately disturbed, and disturbed) defined by the appropriate choice of the model parameters to obtain data for Figure 1. We did not try to analyze any effects of substorm activity, although several observational and modeling studies have noted the existence of the displacements of conjugate points dependent on sub-storms [Stenbaek-Nielsen et al., 1972; Frank and Sigwarth., 2003; Motoba et al., 2010; Saita et al., 2011; Østgaard et al., 2011]. We presented other results (Figures 2-5) for the moderately disturbed period of the magnetic field lines starting at $70^{\circ}$ latitudes, since these lines exhibit latitudinal differences in the southern hemisphere and are closed for both winter and summer times.

[36] It was found that while the latitudinal difference is of the order of $2^{\circ}$, the longitudinal difference can be as large as $30^{\circ}$. This is in agreement with the previous studies, where it was demonstrated that auroral features may not always be conjugate [Belon et al., 1969; Stenbaek-Nielsen et al., 1972; Sato et al., 2004] and the longitudinal displacements are more pronounced [Sato et al., 1998; Minatoya et al., 1996; Frank and Sigwarth., 2003; Østgaard et al., 2004].

[37] The magnitudes of the differences depend on the dipole tilt angle, solar wind dynamic pressure, presence of IMF $B_{y}$, and the sign of IMF $B_{z}$. The dipole tilt angle is an important factor of the relative displacement of the auroral features between the hemispheres [Ostgaard et al., 2005]. Saita et al. [2011] interpreted the role of the dipole tilt as being the result of the seasonally dependent fieldaligned current (FAC) intensity in the pre-midnight sector. According to Ohtani et al. [2005, 2009], the dayside FAC is more intense in summer than in winter, and for FAC in the pre-midnight sector, the opposite. If there is a stronger FAC in winter, the two pairs of FACs give a larger magnetic field variation in winter. This is in agreement with our Figure 1, where the magnetic field lines come to the southern hemisphere at higher latitudes on the dayside and at lower latitudes on the nightside in northern winter and vice versa 
in summer. Laundal and Østgaard [2009] reported that the displacement of auroras in the opposite hemispheres is due to the seasonal effects.

[38] Solar wind dynamic pressure has not been considered as a critical parameter when studying magnetic conjugacy, most probably because any solar wind pressure is thought to apply evenly on both hemispheres. We found that the magnetic mapping on the nightside magnetosphere is much more affected by large solar wind dynamic pressure (Figure 3 ) than the dayside magnetosphere: the maximum latitudinal difference of $\pm 2^{\circ}$ is present only around midnight for large values of $P_{s w}(>10 \mathrm{nPa})$, and with no dependence of longitudinal difference on $P_{s w}$ magnitude on the dayside, it can be as large as $10^{\circ}$ at 22 and 02 MLT. When large solar wind dynamic pressure is present, it compresses the magnetosphere and the tail becomes inflated. If IMF $B_{z}$ is negative, reconnection is going on, and magnetic flux is accumulated in the tail. The tail current is strong under the compression. As a result, the magnetic field in the lobes increases, the current in the tail increases, and the asymmetric configuration forms. Due to this, the latitudinal difference also increases. Here such a configuration is discussed that is already asymmetric due to nonzero dipole tilt $\left( \pm 33^{\circ}\right)$. The effect of nonzero tilt is that it shifts the plasma sheet (and, therefore, the tail current) from the equatorial plane of the Earth's magnetic dipole. Thus, in the absence of tail current, there would be symmetric north-south mapping of the field lines. The strong tail current will move tailward the end of the field line in the neutral sheet (and away from the equatorial plane of the Earth's dipole) and create the asymmetry in ionospheric points of a field line in two hemispheres. The stronger the tail current, the larger the difference in ionospheric foot points. The longitudinal difference is also present with a day-night asymmetry.

[39] It is well known that the interplanetary magnetic field orientation controls the magnetospheric morphology. In particular, when the IMF $B_{y}$ component penetrates into the magnetotail, the tail configuration twists [Cowley, 1981]. Since the magnetic field lines are distorted by the twisted magnetotail, the conjugate points will be displaced. Our findings (Figure 4) that the latitudinal differences reach about $2^{\circ}$ and longitudinal differences are about $20^{\circ}$ at dawn and dusk MLTs for large dipole tilt angles and large IMF $B_{y}$ values (-15 and $15 \mathrm{nT})$ are in agreement with previous studies [Ostgaard et al., 2004, 2005]. At the same time, the important fact found in this study is that the asymmetry of conjugate points is present due to nonzero values of IMF $B_{y}$ but there is no real dependence on the magnitude of IMF $B_{y}$. The influence of IMF $B_{z}$ on the interhemispheric conjugacy depends very much on the sign of IMF $B_{z}$ but not much on its magnitude. There are only very small variations when IMF $B_{z}$ is positive, but the dependence is very pronounced when IMF $B_{z}$ is $<-2 \mathrm{nT}$ (Figure 5). Only IMF $B_{z}$ gives large latitudinal differences in two hemispheres (3 or $4^{\circ}$ in absolute values around 20 and 05 MLT) due to intense dayside reconnection for negative $B_{z}$ values. This leads to strong tail current enhancement and north-south asymmetry for nonzero tilts. The effect is similar to that of the dynamic pressure increase, but here it is the result of magnetic flux increase due to the dayside reconnection, which is more efficient than the flux increase due to magnetospheric contraction under a pressure pulse.
[40] In addition to the standard T02 parameters, a notable dependence on the non-radial solar wind flow was revealed by rotating the model tail current sheet above and below the Sun-Earth line. The importance of taking into account the solar wind flow direction was confirmed by the analysis of the observed and modeled magnetic conjugacy of Tjörnes (Iceland) and Syowa (Antarctica) observatories, which detected two similar auroras simultaneously with allsky TV cameras. For these conjugate observations, Sato et al. [2005] found that none of the T96 and T02 Tsyganenko models could reproduce the observed displacement, when the observed solar wind and IMF parameters were used as input. However, during this event the $V_{z}$ component of the solar wind velocity was not close to zero but about $50 \mathrm{~km} / \mathrm{s}$. When any of $V_{y}$ and $V_{z}$ components are not close to zero, even small deviations of the tail location relative to the SunEarth line can cause notable variations in the magnetic field. This effect must be taken into account when studying the magnetic conjugacy. With the inclusion of the tail tilt as a modification to the T02 model, we can explain the latitudinal locations of the conjugate auroral observations.

[41] On the other hand, we obtained the longitudinal displacement of the observed auroras 2 times smaller than that observed. There are two possible interpretations:

[42] 1. We modeled the foot points with the T02 model which gives only an averaged magnetic configuration and does not have sub-storm variations included which should be present. At the same time, it is rather hard to estimate what could be the locations of the foot prints if we have a better model. For example, it may be that stretching of field lines will increase the difference due to the solar wind non-radial flow in the same direction. The field lines will cross the equator at larger radial distances where neutral sheet is rotated further away the $X_{\mathrm{GSM}}$ axis. It may result in the longitudinal displacement comparable to observations.

[43] 2. During the conjugacy event reported by Sato et al. [2005], the IMF $B_{x}$ decreased from $-1.0 \mathrm{nT}$ to $-2.5 \mathrm{nT}$. This can have the same effect on the footprints in the southern hemisphere as the presence of positive $V_{z}$ and result in the increase of the model longitudinal (and latitudinal) shift [Kubyshkina et al., 2012].

[44] The problem of the inter-hemispheric conjugacy still remains open since there is only very few possibilities to monitor the real conjugacy with simultaneous observations in both hemispheres. The existing magnetic field models are not able to give the true conjugate points with high accuracy. There is a good number of additional factors which may change the given field line configuration including the hemispherically asymmetric strong field-aligned currents [Kaufmann et al., 1990; Sato et al., 1998], or inter-hemispheric currents [Benkevich et al., 2000; Stenbaek-Nielsen and Otto, 1997], or even ionospheric currents and conductivities. Nevertheless, the main properties of the behavior of the magnetic field lines can be derived from the solar wind and IMF input to the model. Our study indicates the regular displacements of conjugate points due to the presence of the asymmetries in the model magnetic field. The model can be not completely accurate but the real asymmetry exists.

[45] The main conclusions obtained are given below:

[46] 1. Latitudinal and longitudinal differences of conjugate points and their dependence on the dipole tilt angle: 
(a) For quiet conditions, only slight deviations in latitudes at which the magnetic field lines start from the northern hemisphere and to which they come down to the southern hemisphere are present for higher latitudes $\left(75^{\circ}\right)$ for dipole tilt angles corresponding to winter $\left(-33^{\circ}\right)$ and summer $\left(+33^{\circ}\right)$.

(b) For moderately disturbed conditions, the magnetic field lines starting at $75^{\circ}$ latitudes on the dayside in the northern hemisphere do not come at all to the southern hemisphere regardless of the tilt angle, and for disturbed periods, this happens to the magnetic field lines starting at as low as $60^{\circ}$.

(c) The latitudinal differences exhibit the reversed asymmetry depending on the season: The magnetic field lines come to the southern hemisphere at higher latitudes on the dayside and at lower latitudes on the nightside in winter and vice versa in summer.

(d) While peak magnitudes of latitudinal differences for magnetic field lines starting at $70^{\circ}$ in the northern hemisphere during moderately disturbed conditions are only about $2^{\circ}$, the longitudinal differences are as large as $30^{\circ}$ at dawn and dusk MLTs for large dipole tilt angles.

[47] 2. Latitudinal and longitudinal differences of conjugate points and their dependence on the solar wind and IMF parameters:

(a) Latitudinal differences reach about $2^{\circ}$, whereas longitudinal differences are $20^{\circ}$ at dawn and dusk MLTs for large dipole tilt angles during high solar wind dynamic pressure (16-20 $\mathrm{nPa})$ and large IMF $B_{y}$ values $(-15$ and $15 \mathrm{nT})$.

(b) Magnetic conjugacy in the nightside magnetosphere is much more affected by large solar wind dynamic pressure: the maximum latitudinal difference of $\pm 2^{\circ}$ is present only around midnight for large values of $P_{s w}$ $(>10 \mathrm{nPa})$, and with no dependence of the longitudinal difference on $P_{s w}$ magnitude on the dayside, it can be as large as $10^{\circ}$ at 22 and 02 MLT.

(c) The asymmetry of conjugate points is present due to nonzero values of IMF $B_{y}$, but there is only a weak dependence on the magnitude of IMF $B_{y}$.

(d) The influence of IMF $B_{z}$ on the inter-hemispheric conjugacy depends very much on the sign of the IMF $B_{z}$ but not much on its magnitude. There are only small variations when IMF $B_{z}$ is positive, but the dependence is very pronounced when IMF $B_{z}$ is $<-2 \mathrm{nT}$ (large latitudinal differences in the two hemispheres; 3 or $4^{\circ}$ in absolute values around 20 and 05 MLT).

[48] 3. The rotation of the tail current sheet from the Sun-Earth line by several degrees (from +8 to $-8^{\circ}$ ), which represents the influence of non-radial solar wind flow on the magnetic field configuration, results in latitudinal differences of $1^{\circ}$ and longitudinal differences of $15^{\circ}$ at dawn and dusk MLTs during equinox. Analysis of the magnetic conjugacy of Tjörnes (Iceland) and Syowa (Antarctica) observatories, which detected two similar auroral structures with all-sky TV cameras, confirmed the importance of taking into account the solar wind flow direction when it deviates from radial by more than $1-2^{\circ}$.
[49] Acknowledgments. We thank OMNIWEB data center for IMF and solar wind parameter data, World Data Center C2 for Geomagnetism, Kyoto, for the Dst indices data. N. Ganushkina's work was partly supported by the Academy of Finland. N. Ganushkina gratefully acknowledges the support of her work at the University of Michigan by grants from NASA and NSF. N. Ganushkina and M. Kubyshkina thank the International Space Science Institute in Bern, Switzerland, for their support of an international team on "Resolving Current Systems in Geospace." The work of E. Tanskanen was supported by the Academy of Finland. We thank M. Liemohn for valuable discussions.

\section{References}

Belon, A. E., J. E.Maggs, T. N. Davis, K. B. Mather, N.W. Glass, and G. G. Hughes (1969), Conjugacy of visual auroras during magnetically quiet periods, J. Geophys. Res., 74, 1-28.

Benkevich, L., W. Lyatsky, and L. L. Cogger (2000), Field-aligned currents between conjugate hemispheres, J. Geophys. Res., 105(A12), 27, 727-27,737, doi:10.1029/2000JA900095.

Cowley, S. W. H. (1981), Magnetospheric asymmetries associated with the y-component of the IMF, Planet. Space Sci., 29, 79-96, doi: 10.1016/0032-0633(81)90141-0.

Frank, L. A. (1995), The Visible Imaging System (VIS) for the Polar spacecraft, Space Sci. Rev., 71, 297-328.

Frank, I. A., and J. B. Sigwarth (2003), Simultaneous images of the northern and southern auroras from the Polar spacecraft: An auroral substorm, $J$. Geophys. Res., 108(A4), 8015, doi:10.1029/2002JA009356.

Hones, E. W., Jr., R. D. Zwickl, T. A. Fritz, and S. J. Bame (1986), Structural and dynamical aspects of the distant magnetotail determined from ISEE3 plasma measurements, Planet. Space Sci., 34, 889-901.

Kaufmann, R. L., D. J. Larson, and C. Lu (1990), Mapping and distortions of auroral structures in the quiet magnetosphere, J. Geophys. Res., 95(A6), 7973-7994, doi:10.1029/JA095iA06p07973.

Kubyshkina, M. V., V. A. Sergeev, and N. A. Tsyganenko (2012), Solar wind control of magnetospheric neutral sheet position-Results obtained with adjusted empirical magnetic field models, presented at 11th International Conference on Substorms, Lüneburg, Germany

Laundal, K., and N. Østgaard (2009), Asymmetric auroral intensities in the Earth's Northern and Southern hemispheres, Nature, 460, 491-493, doi: 10.1038 /nature08154

Laundal, K., N. Østgaard, K. Snekvik, and H. U. Frey (2010), Interhemispheric observations of emerging polar cap asymmetries, J. Geophys. Res., 115, A07230, doi:10.1029/2009JA015160.

Mende, S. B., et al. (2000), Far ultraviolet imaging from the IMAGE spacecraft. 2. Wideband FUV imaging, Space Sci. Rev., 91, 271-285.

Minatoya, H., N. Sato, T. Saemundsson, and T. Yoshino (1996), Large displacements of conjugate auroras in the midnight sector, J. Geomag. Geoelectr., 48, 967-975.

Motoba, T., K. Hosokawa, N. Sato, A. Kadokura, and G. Bjornsson (2010), Varying interplanetary magnetic field $B_{y}$ effects on interhemispheric conjugate auroral features during a weak substorm, J. Geophys. Res., 115, A09210, doi:10.1029/2010JA015369.

Motoba, T., K. Hosokawa, A. Kadokura, and N. Sato (2012), Magnetic conjugacy of northern and southern auroral beads, Geophys. Res. Lett., 39, L08108, doi:10.1029/2012GL051599.

Ono, T. (1987), Temporal variation of the geomagnetic conjugacy in Syowa-Iceland pair, Mem. Natl. Inst. Polar Res., Special Issue, 48, 46-57.

Ohtani, S., G. Ueno, T. Higuchi, and H. Kawano (2005), Annual and semiannual variations of the location and intensity of large-scale field-aligned currents, J. Geophys. Res., 110, A01216, doi:10.1029/2004JA010634.

Ohtani, S., S. Wing, G. Ueno, and T. Higuchi (2009), Dependence of premidnight field-aligned currents and particle precipitation on solar illumination, J. Geophys. Res., 114, A12205, doi:10.1029/2009JA014115.

Saita, S., et al. (2011), Displacement of conjugate points during a substorm in a global magnetohydrodynamic simulation, J. Geophys. Res., 116, A06213, doi:10.1029/2010JA016155.

Sato, N., T. Nagaoka, K. Hashimoto, and T. Saemundsson (1998), Conjugacy of isolated auroral arcs and nonconjugate auroral breakups, $J$. Geophy. Res., 103, 11,641-11,652.

Sato, N., D. M. Wright, C. W. Carlson, Y. Ebihara, M. Sato, T. Saemundsson, S. E. Milan, and M. Lester (2004), Generation region of pulsating aurora obtained simultaneously by the FAST satellite and a Syowa-Iceland conjugate pair of observatories, J. Geophys. Res., 109, A10201, doi:10.1029/2004JA010419.

Sato, N., A. Kadokura, Y. Ebihara, H. Deguchi, and T. Saemundsson (2005), Tracing geomagnetic conjugate points using exceptionally similar synchronous auroras, Geophys. Res. Lett., 32, L17109, doi:10.1029/ 2005 GL023710. 


\section{GANUSHKINA ET AL.: INTERHEMISPHERIC MAGNETIC CONJUGACY}

Shue, J.-H., J. K. Chao, H. C. Fu, C. T. Russell, P. Song, K. K. Khurana and H. J. Singer (1997), A new functional form to study the solar wind control of the magnetopause size and shape, J. Geophys. Res., 102(A5), 9497-9511, doi:10.1029/97JA00196.

Stenbaek-Nielsen, H. C., T. N. Davis, and N. W. Glass (1972), Relative motion of auroral conjugate points during substorms, J. Geophys. Res., $77,1844-1858$.

Stenbaek-Nielsen, H. C., and A. Otto (1997), Conjugate auroras and the interplanetary magnetic field, J. Geophys. Res., 102(A2), 2223-2232, doi: 10.1029/96JA03563.

Tanaka, T., A. Nakamizo, A. Yoshikawa, S. Fujita, H. Shinagawa, H. Shimazu, T. Kikuchi, and K. K. Hashimoto (2010), Substorm convection and current system deduced from the global simulation, J. Geophys. Res., 115, A05220, doi:10.1029/2009JA014676.

Tsyganenko, N. A., and A. V. Usmanov (1982), Determination of the magnetospheric current system parameters and development of experimental geomagnetic field models based on data from IMP and HEOS satellites, Planet. Space Sci., 30, 985-998.

Tsyganenko, N. A. (1995), Modeling the Earth's magnetospheric magnetic field confined within a realistic magnetopause, J. Geophys. Res., 100, 5599-5612.

Tsyganenko, N. A., S. B. P. Karlsson, S. Kokubun, T. Yamamoto, A. J. Lazarus, K. W. Ogilvie, C. T. Russell, and J. A. Slavin (1998), Global configuration of the magnetotail current sheet as derived from Geotail, Wind, IMP 8 and ISEE 1/2 data, J. Geophys. Res., 103(A4), 6827-6841, doi:10.1029/97JA03621.

Tsyganenko, N. A. (2002a), A model of the near magnetosphere with a dawn-dusk asymmetry: 1. Mathematical structure, J. Geophys. Res., 107, SMP 12-1, CiteID 1179, doi:10.1029/2001JA0002192001.

Tsyganenko, N. A. (2002b), A model of the near magnetosphere with a dawn-dusk asymmetry: 2. Parameterization and fitting to observations, J. Geophys. Res, 107, SMP 10-1, CiteID 1176, doi:10.1029/ 2001JA000220.

Tsyganenko, N. A., H. J. Singer, and J. C. Kasper (2003), Storm-time distortion of the inner magnetosphere: How severe can it get? J. Geophys. Res., 108(A5), 1209, doi:10.1029/2002JA009808.

Tsyganenko, N. A., and D. H. Fairfield (2004), Global shape of the magnetotail current sheet as derived from Geotail and Polar data, J. Geophys. Res., 109, A03218, doi:10.1029/2003JA010062.

Tsyganenko, N. A., and M. I. Sitnov (2005), Modeling the dynamics of the inner magnetosphere during strong geomagnetic storms, J. Geophys. Res., 110, A03208, doi:10.1029/2004JA010798.

O. I. Yagodkina, D. Sibeck, K. Liou, and C.-I. Meng (2001), Aurora conjugacy during substorms: Coordinated Antarctic ground and Polar Ultraviolet observations, J. Geophys. Res., 106, 24,579-24,591.

Østgaard, N., S. B. Mende, H. U. Frey, T. J. Immel, L. A. Frank, J. B. Sigwarth, and T. J. Stubbs (2004), Interplanetary magnetic field control of the location of substorm onset and auroral features in the conjugate hemisphere, J. Geophys. Res., 109, A07204, doi:10.1029/2003JA 010370 .

Østgaard, N., N. A. Tsyganenko, S. B. Mende, H. U. Frey, T. J. Immel, M. Fillingim, L. A. Frank, and J. B. Sigwarth (2005), Observations and model predictions of substorm auroral asymmetries in the conjugate hemispheres, Geophys. Res. Lett., 32, doi:10.1029/ 2004 GL022166.

Østgaard, N., K. M. Laundal, L. Juusola, A. Åsnes, S. E. Håland, and J. M. Weygand (2011), Interhemispherical asymmetry of substorm onset locations and the interplanetary magnetic field, Geophys. Res. Lett., 38, L08104, doi:10.1029/2011GL046767.

Viljanen, A., and E. Tanskanen (2012), High-latitude magnetic fields and their time derivatives: interhemispheric similarities, Earth, Planets and Space, in press. 